\title{
An ISDS-Based Initiative for Conventions for Biosurveillance Data Analysis Methods
}

\author{
Michael Coletta ${ }^{4}$, Howard Burkom*1, Jeffrey Johnson² and Wendy Chapman ${ }^{3}$ \\ ${ }^{1}$ Johns Hopkins Applied Physics Laboratory, Laurel, MD, USA; ${ }^{2}$ San Diego County Health and Human Services Agency, San Diego, \\ CA, USA; ' ${ }^{3}$ nniversity of California San Diego, San Diego, CA, USA; ${ }^{4}$ National Association of County and City Health Officials, \\ Washington, DC, USA
}

\section{Objective}

The panel will present the problem of standardizing analytic methods for public health disease surveillance, enumerate goals and constraints of various stakeholders, and present a straw-man framework for a conventions group.

\section{Introduction}

Twelve years into the 21 st century, after publication of hundreds of articles and establishment of numerous biosurveillance systems worldwide, there is no agreement among the disease surveillance community on most effective technical methods for public health data monitoring. Potential utility of such methods includes timely anomaly detection, threat corroboration and characterization, follow-up analysis such as case linkage and contact tracing, and alternative uses such as providing supplementary information to clinicians and policy makers.

Several factors have impeded establishment of analytical conventions. As immediate owners of the surveillance problem, public health practitioners are overwhelmed and understaffed. Goals and resources differ widely among monitoring institutions, and they do not speak with a single voice. Limited funding opportunities have not been sufficient for cross-disciplinary collaboration driven by these practitioners. Most academics with the expertise and luxury of method development cannot access surveillance data. Lack of data access is a formidable obstacle to developers and has caused talented statisticians, data miners, and other analysts to abandon the field. The result is that older research is neglected and repeated, literature is flooded with papers of varying utility, and the decision-maker seeking realistic solutions without detailed technical knowledge faces a difficult task.

Regarding conventions, the disease surveillance community can learn from older, more established disciplines, but it also poses some unique challenges. The general problem is that disease surveillance lies on the fringe of disparate fields (biostatistics, statistical process control, data mining, and others), and poses problems that do not adequately fit conventional approaches in these disciplines.

In its eighth year, the International Society of Disease Surveillance is well positioned to address the standardization problem because its membership represents the involved stakeholders including progressive programs worldwide as well as resource-limited settings, and also because best practices in disease surveillance is fundamental to its mission. The proposed panel is intended to discuss how an effective, sustainable technical conventions group might be maintained and how it could support stakeholder institutions.

\section{Methods}

Members of a Technical Conventions Group would have experience and dedication to advancing the science of disease surveillance. Primary functions would include:

1. Specify and communicate technical problems facing professionals involved in routine monitoring of population health. Alternative use applications would also be considered, such as the use of epidemiological findings to inform clinical diagnoses.

2. Independently evaluate the utility of proposed analytical solutions to well-defined problems in public health surveillance and confer approval or certification, perhaps on several levels, such as whether results can be replicated with shareable data. Approved solutions might be restricted to commonly available software such as the R language or Microsoft EXCEL.

3. Facilitate sharing of tools and methodologies to evaluate methods and to visualize their results

The framework to be discussed in the proposed panel would be a means of keeping open lines of collaboration and idea-sharing. Overcoming obstacles toward this goal is worthy of a conference panel discussion whether or not it concludes that a conventions group is a viable approach.

\section{Results}

Three 15-minute panelist talks are proposed:

1. Background: in-depth description of the dimensions of the problem above

2. Constraints facing public health practitioners and requirements for practical analytic tools

3. Strawman conventions group: role, logistics, inclusiveness, methods of communicating with stakeholders and related organizations and producing/disseminating output.

For the 45 minutes of discussion, the first 15-20 will invite reactions to the first two talks to sharpen the scope of the effort. The remainder of the session will cover the advisability, feasibility, and logistics of an ISDS-based conventions group, and modify the strawman group concept.

\section{Keywords}

Standards; Data Analysis; Statistical Algorithms; Certification

*Howard Burkom

E-mail: howard.burkom@jhuapl.edu 